\title{
Hyperdense Basilar Artery Sign
}

\author{
Hirofumi Goto ${ }^{1}$, Tatsuya Tanaka ${ }^{2}$, Masahito Yoshida ${ }^{3}$ and Nobuaki Momozaki ${ }^{2}$
}

Key words: basilar artery occlusion, hyperdense basilar artery sign

(Intern Med 54: 991-992, 2015)

(DOI: 10.2169/internalmedicine.54.3338)
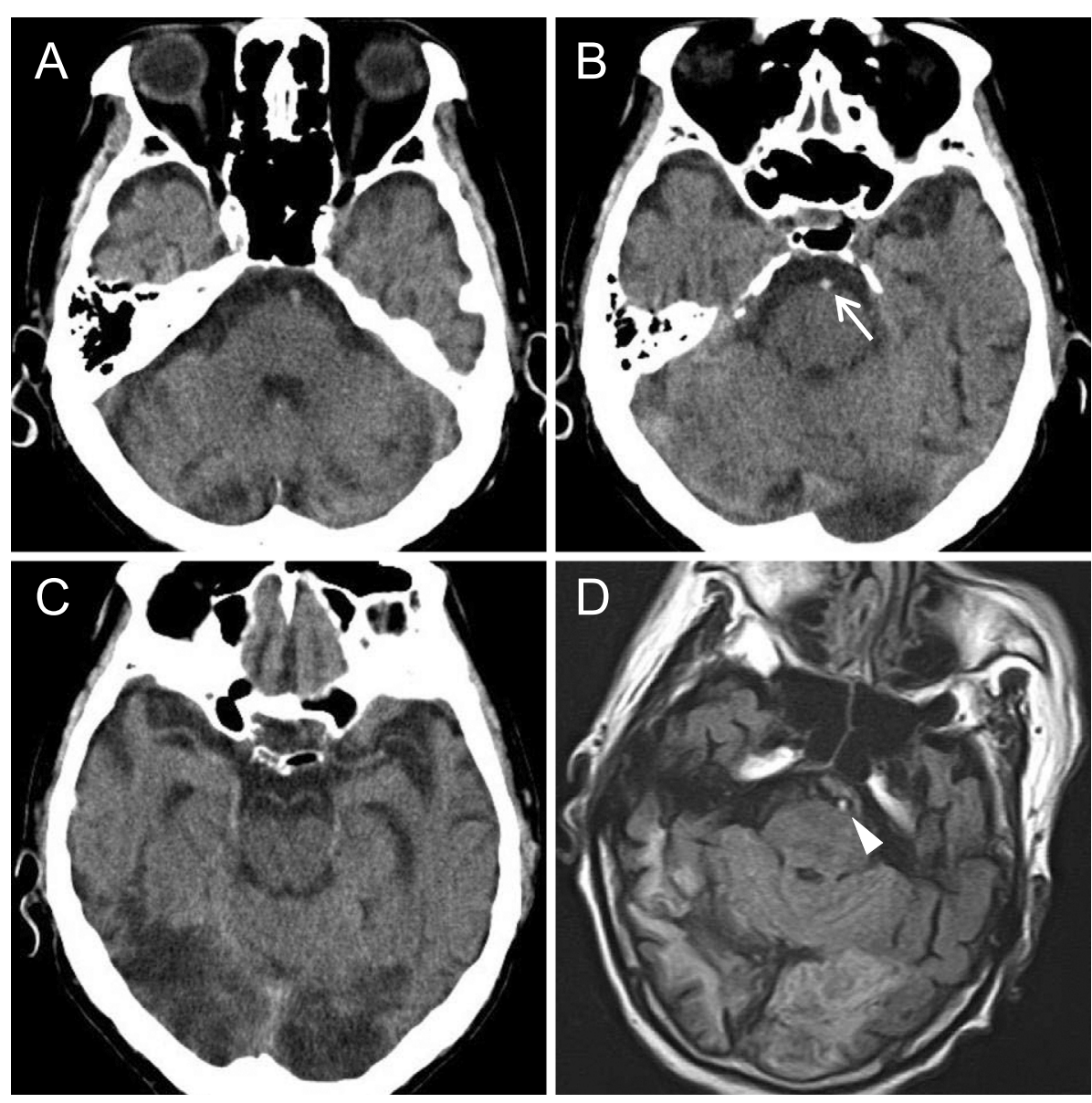

Picture 1.

A 93-year-old man with hypertension presented with a sudden disturbance of consciousness. A neurological examination revealed a loss of consciousness with a Glasgow Coma Scale score of 6 (E1V1M4), ataxic respiration and bilateral miosis. Adjacent slices from a brain computed tomography performed three hours after the onset (AO) showed a hyperdense change of the basilar artery (BA) (Pic- ture $1 \mathrm{~A}-\mathrm{C}$, arrow), thus indicating a hyperdense basilar artery (HDBA) sign (1). Brain fluid-attenuated inversion recovery images obtained four hours AO demonstrated a hyperintense signal of the BA, suggesting a complete occlusion due to an embolus or thrombus (Picture 1D, arrow head) (2). Brain diffusion-weighted images (Picture 2) revealed fresh infarctions of the bilateral cerebellum, pons and

${ }^{1}$ Department of Neurology, Imari Arita Kyouritsu Hospital, Japan, ${ }^{2}$ Department of Neurosurgery, Imari Arita Kyouritsu Hospital, Japan and ${ }^{3}$ Department of Emergency Medicine, Imari Arita Kyouritsu Hospital, Japan

Received for publication May 23, 2014; Accepted for publication September 10, 2014

Correspondence to Dr. Hirofumi Goto, goto-hirofumi@imari-arita-hp.or.jp 

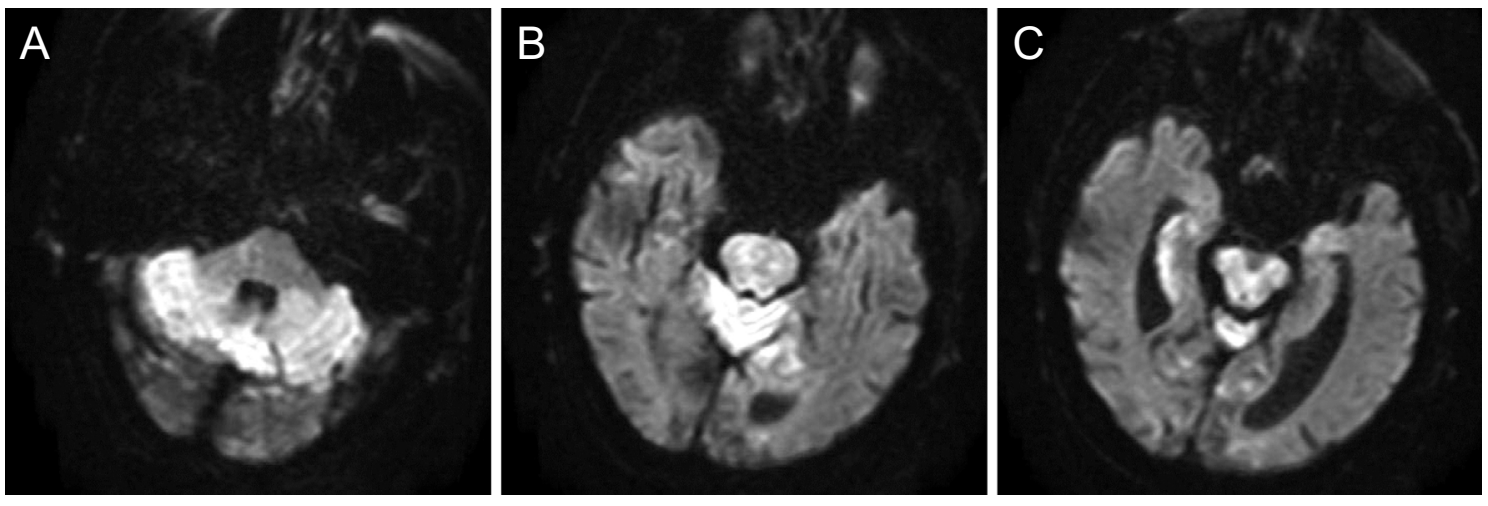

Picture 2.

midbrain. The patient died from respiratory failure seven hours AO. The presence of a HDBA sign therefore suggests the occurrence of both basilar artery occlusion and brainstem infarction and may predict a poor prognosis for such patients (1).

The authors state that they have no Conflict of Interest (COI).

\section{References}

1. Goldmakher GV, Camargo EC, Furie KL, et al. Hyperdense basilar artery sign on unenhanced CT predicts thrombus and outcome in acute posterior circulation stroke. Stroke 40: 134-139, 2009.

2. Toyoda K, Ida M, Fukuda K. Fluid-attenuated inversion recovery intraarterial signal: an early sign of hyperacute cerebral ischemia. AJNR Am J Neuroradiol 22: 1021-1029, 2001.

(C) 2015 The Japanese Society of Internal Medicine http://www.naika.or.jp/imonline/index.html 